\title{
The 2014 Moreton Lecture: A Rebuttal
}

Gitasree Borthakur, MD, William D. Kerridge, MD, Zachary Ballenger, MD, Richard B. Gunderman, MD,

$\mathrm{PhD}$

Porter's Value-Based Health Care: The Prognosis

At the 2014 ACR AMCLC, the Moreton Lecture was delivered by Michael Porter, Bishop William Lawrence University Professor at the Harvard Business School. Author of 18 books and 6-time winner of the McKinsey Award for best Harvard Business Review article of the year, Porter is often hailed as the world's foremost authority on business strategy and the most cited author in business and economics worldwide. Recently, Porter has turned his attention to health care, including a 2007 book titled Redefining Health Care (coauthored with Elizabeth Olmsted Teisberg) and a 2009 New England Journal of Medicine article, "A Strategy for Health Care Reform-Toward a Value-Based System."

Porter's Moreton Lecture drew heavily from his 2013 Harvard Business Review article "The Strategy That Will Fix Health Care." In it, Porter and his coauthor Thomas H. Lee suggest that the key to fixing health care is to increase the value it provides to patients. Value may be defined as the ratio of quality to cost, so the value of health care can be improved by increasing quality or lowering cost. On the surface, measuring costs sounds easy, but they often turn out to be rather slippery, in terms of both magnitude and the issue of who bears them. Quality, of course, is much more difficult to define, let alone measure, and different health care constituencies-patients, health professionals, hospitals, and payers - often look at quality quite differently.

This is the author's manuscript of the article published in final edited form as:

Borthakur, G., Kerridge, W. D., Ballenger, Z., \& Gunderman, R. B. (2015). The 2014 Moreton Lecture: A Rebuttal. Journal of the American College of Radiology, 12(7), 676-677.

http://doi.org/10.1016/i.jacr.2014.12.002 
At the core of Porter's value-based approach is a difficulty. On one hand, he suggests that US health care providers need to replace their traditional volume- and margin-based vision of success with one focused on value. On the other hand, however, he soon finds himself arguing that one of the keys to providing value in health care is to centralize many health care services in large units that provide highvolume services. As long as every facility attempts to provide virtually all health services, he suggests, each one can see only a relatively small number of cases of a particular type. By encouraging health care facilities to specialize in particular service lines, much larger numbers of particular diagnoses can be concentrated in a single place, fostering enhanced quality and efficiency.

The key concept here is one Porter labels the integrated practice unit (IPU). Porter adduces the example of an IPU for diabetes, which would provide the "full care cycle" for patients with diabetes, including disease-specialist physicians, nurses, and other health professionals who focus on the disease and its complications. Porter specifically mentions the need for eye and kidney care. Here, one of the weaknesses of applying a business consulting background to health care becomes apparent. Although Porter is the world's authority on business strategy, he seems to lack expertise in medicine, evidently failing to grasp that "full-cycle" diabetes care would also require a variety of other medical services, including endocrinologic, cardiovascular, neurologic, and infectious disease care.

Before long, what starts out as a dedicated IPU specializing in diabetes care begins to bear a striking resemblance to a general hospital. Simply put, the afflictions associated with diabetes are not a distinct subset of medical problems. In fact, long-term diabetes often takes a toll on virtually every organ system in the human body. Moreover, many patients with diabetes also have a number of comorbid conditions, including hypertension, atherosclerosis, degenerative joint disease, and impaired wound healing. Theoretically, a health care facility specializing in diabetes sounds like a great idea, but to health professionals with substantial experience caring for such patients, it is difficult to conceive of such a facility as anything much less than a full-service hospital. 
There is nothing novel in Porter's second strategy for fixing health care, namely, measuring outcomes. However, Porter does seek to put a distinctive spin on this recommendation by suggesting that the outcomes that get measured are the ones that matter to patients. What is not yet clear, however, is whether the large expenditures required to achieve interoperability among different health care information systems will be adequately compensated by improvements in the quality of outcome assessments. Some of the most widely cited outcomes measures, such as life expectancy and infant mortality, are imprecise and in many cases positively misleading, at least in grading health care systems. In other words, we do not yet have strong evidence that assessing outcomes more carefully will improve the value of health care.

Porter follows a well-trodden path in recommending the bundling of health care payments. The underlying idea is that paying providers for services rendered creates a perverse incentive to render more services, so providers must be made to share the costs of inappropriate service and cost overruns. The problem, however, is that even the best physicians and hospitals cannot control medical outcomes. Even if every person involved in a patient's care performs admirably, outcomes can still be poor, and such outcomes often generate substantial extra costs. Moreover, bundling assumes that all patients carrying a particular diagnosis or needing a particular treatment can be lumped together, but patients often turn out to differ from one another in important respects.

Porter argues that we need integrated delivery systems, by which he means reducing the degree of overlap or redundancy among health care providers and facilities in the same geographic region. For example, large academic centers might focus on high-tech services while discontinuing more routine ones. Such an approach might work in a single-payer health care system, but as long as hospitals and health systems are competing with one another, such a scenario appears problematic. This stems in part from the fact that Porter's proposal would reduce competition among health service providers, reducing 
incentives to increase quality and lower cost. At least a present, competition plays an important role in motivating providers to enhance value.

Porter also evinces great faith in IT. He calls for the development of a system that would be patient centered, use common data definitions, encompass all types of data, include expert systems for every medical condition, make it easy to extract information, and extend beyond particular hospitals and health systems. Although such goals are difficult to argue against in the abstract, they are likely to prove devilishly difficult to achieve in practice. And even if such a system could be constructed, it is important to acknowledge the wide chasm that still separates a robust information system from the professional wisdom and judgment required to take good care of patients. Good information is valuable, but it is no substitute for a strong patient-physician relationship.

And here one of the most important limitations of Porter's strategy comes fully into view. Ultimately, Porter focuses on economic and technological parameters of health care, such as value and interoperability. By contrast, he evinces surprisingly little interest in the human relationships on which health care always depends. For example, a patient who needs to visit 3 or 4 different IPUs for care of multiple conditions is likely to suffer from a lack of continuity of care. To be sure, information may be readily accessible at each facility, but who will coordinate the patient's care, overseeing all aspects of service and building a deep and enduring relationship with the patient? Great health care is really about great medicine, and great medicine is built on great relationships.

Will radiologists who attend carefully to Porter's message heed his advice? Will they adopt strategies designed around his vision for the future of health care and thereby prosper in the years to come? The short answer: no one knows. Porter is an internationally heralded guru of business strategy, and we are just a group of ordinary physicians working on health care's frontlines. That being said, however, Porter's vision seems very far removed from our daily work as health professionals. Occasionally it seems deeply at odds with our sense of what is most real and important about medicine 
and the care of patients. For these reasons, we are inclined to bet against him. Although his argument is ingenious, we believe that it is also largely wrong 